\title{
Collaboration and Decision Making in Crisis Situations
}

Adriana S. Vivacqua

Universidade Federal do

Rio de Janeiro

Rio de Janeiro, RJ, BRAZIL

avivacqua@dcc.ufrj.br

Ana Cristina B. Garcia

Universidade Federal

Fluminense

Rio de Janeiro, RJ, BRAZIL

bicharra@ic.uff.br

\section{José H Canós}

Universitat Politècnica de

València

Valencia, Spain

jhcanos@dsic.upv.es

Permission to make digital or hard copies of part or all of this work for personal or classroom use is granted without fee provided that copies are not made or distributed for profit or commercial advantage and that copies bear this notice and the full citation on the first page. Copyrights for third-party components of this work must be honored. For all other uses, contact the Owner/Author. Copyright is held by the owner/author(s)

CSCW '16 Companion, February 27 - March 02, 2016, San Francisco, CA USA. ACM 978-1-4503-3950-6/16/02.

http://dx.doi.org/10.1145/2818052.2855520

\section{Martina Comes}

tina.comes@uia.no

\section{Vaninha Vieira}

Universidade Federal da

Salvador, BA, Brazil

vaninha@ufba.br

\begin{abstract}
Emergencies are critical situations that demand immediate action to avoid adverse consequences to life and property. Recent events around the world highlight the importance of the theme. A key challenge in

Emergency Management is decision-making under time pressure, with an overload of unconfirmed, uncertain and conflicting information, including the management of many people, with distinct and possibly fluid roles, in different places. Collaboration in these settings is an interesting element, since emergency response generally involves multiple agencies and the public, which, having different views, protocols and priorities, must act in concert to handle the situation. In addition, an increasing amount of virtual information is necessary to inform and manage volunteers. The goal of this workshop is to identify and map the main challenges of collaboration in crisis situations, review current research methods and approaches to address them and address the lack of formal processes, structures, methodologies and tools.
\end{abstract}

\section{Author Keywords}

Emergency management; collaborative decision making; time constraints; improvisation. 


\section{ACM Classification Keywords}

H.5.3. Information interfaces and presentation (e.g., HCI): Computer Supported Cooperative Work; H.4. Information Systems Applications: Decision Support (e.g. MIS) H.1.2. Models and Principles: Human Factors.

\section{Introduction}

Emergency response usually involves multiple agencies and teams working in concert to mitigate effects of an unexpected situation. Collaboration is necessary for effective emergency management, but is also a challenge, given the nature of the task [9][1]. Despite existing protocols, time pressure and the high variability of the environment make it hard to establish effective collaboration between agencies, respondents and the public [1]. Very frequently, protocols are not directly applicable and different rules, norms and goals come into play when different stakeholders are brought together. Differences in organizational culture, chain of command, language, goals and practices also come into play in these situations, rendering emergency management even harder.

Frequent changes in the state of environment, the speed at which the event unfolds and the stressful situation make it hard for participants to develop appropriate situation awareness, or grasp what is going on. Respondents must be prepared to work with information that may be incomplete, inaccurate or in conflict with other information. Thus, disaster response will require improvisation and creativity, elements considered integral to resilience [6]. Traditional process models of coordination provide limited flexibility since they usually take into account only a small number of variations and exceptions, so participants have little freedom to decide what should be done at all times.

Recent approaches inspired in flexible case

management (e.g., [3], [2]) may provide solutions for managing flexible and knowledge-intensive processes.

Decision making is also harder in crisis scenarios than in more controlled situations due to a number of factors. Frequent changes in the situation, time constraints (decisions must be made quickly) and unexpected states or outcomes of these decisions are some of the elements that challenge effective decision making in these settings. These challenges are compounded when multiple actors or agencies with different points of view, goals and protocols are involved in these decisions.

Frequently, emergency management will also involve the general public and volunteers, particularly in events with longer lasting effects (e.g., environmental crisis). In these situations, additional effort is needed to manage these individuals, and to ensure they have the information they need and are properly directed. The public and volunteers can also provide information, from their points of view, as events unfold, functioning as an alternative source of data to be added to official ones. In this type of situations, questions regarding the quality of the information being provided will almost certainly arise, as will issues of merging these data with that from other sources.

This creates an interesting setting for research: decision making and collaboration in crisis situations seem to follow different rules from other situations. In order to build better emergency support tools, we need a better understanding of decision making and collaboration in these conditions. Previous work has 
identified some of the challenges to collaboration in emergency situations, particularly when multiple agencies are involved. These include communicating with each other, establishing and maintaining situation awareness of all parties and creating common ground, so that organizations may understand each other [1]. In addition to that, communication with the public and leveraging crowd information also factor in.

While some research has been conducted in this area (e.g., [4], [5], [7], [8]), we feel there is still much to explore. With this workshop, we hope to attract attention to the topic and get new insights from researchers in the CSCW community. The workshop is geared towards both practitioners and researchers, and has, as its main goal, the establishment of a research agenda in this topic. Some of the questions of interest, and that need to be addressed are:

- How are different protocols and norms handled in interagency collaboration? Can they be trusted to work? How do they affect communication between partners?

- How does time affect decision making and collaboration? Can technology help decisions be made quicker?

- Does hierarchy come into play? Is there a chain of command? How can it be established in a crisis situation? How is trust established between partners in this type of environment?

- What is the involvement of the public? Is it possible to use knowledge generated by the general population? Can this knowledge be trusted? To what extent? Is it possible to engage the public in more active ways without putting them at risk?
- What level of uncertainty can be expected? How does this affect crisis response? How does it affect collaboration? Can technology help deal with this issue?

- Should a history of decisions made and the factors that influence them be kept? Can a line of rationale be established? Can solutions be reused in other settings?

- What methods exist for dealing with improvisation in processes? Can these be applied? What can we learn from other disciplines?

- Can the public contribute more than information? Can they assist in the efforts? How can they be involved and coordinated?

- How is work different in "high alert" environments such as hospitals, control rooms, etc. are emergencies handled differently in these settings than in crisis situations? What can we learn from them?

Different types of studies can shed light into some of these questions:

- Domain studies: studies that characterize the domain, creating a better understanding of the inherent problems. Studies of other domains that exhibit similar characteristics, from which parallels might be drawn are also welcome.

- Methods and techniques proposals: methods, tools and techniques that may be applicable to the problems at hand, even though they may not have been applied to the same domain.

- Cases or deployment studies: applications of technology to real life situations, their consequences and lessons learned. 
Our goal is to encourage discussion and debate, to foster interaction between participants and to spark the generation of new ideas. At the end, the workshop will generate a consolidated report of the discussions, proposed approaches and techniques that could be useful to drive further research. To reach this goal, we will split into subgroups to brainstorm and discuss, looking to identify and map relationships in approaches, gaps and opportunities for research.

\section{Activities and Goals}

The main goal of this workshop is to generate a better understanding of the main challenges of collaboration in crisis situations. Questions regarding the establishment of relations, leadership, structure, decision making, and prioritization of tasks and goals are relevant to our workshop. These findings will then inform later studies and should generate a consolidated report or paper summarizing them.

In an initial moment, participants will be invited to present their work and reflect on each other's work. In a second stage, real life cases will be provided for the group to consider and discuss, this will be done in 4-5 breakout groups (depending on the number of participants). In a final stage, the groups will share their considerations and consolidate them in one model. We are also considering inviting an expert in the field to give a presentation.

\section{Background of Organizers}

Adriana S. Vivacqua is Assistant Professor at the Computer Science Department at the Federal University of Rio de Janeiro (UFRJ). Her research focuses on awareness in collaboration and intelligent human computer interaction, and she has published several papers in these areas. She is a researcher in the Reference Center in Disasters and in the Reference Center in Big Data (CRBD), at UFRJ. She has been the recipient of a Young Researcher Award of the State of Rio de Janeiro twice (2012-2015 and 2015-2018) and holds a productivity grant from the Ministry of Science and Technology (CNPq). She has organized conferences and workshops, is currently posters chair for ISCRAM 2016 and ACM GROUP 2016, and is participating in the organization of CSCW 2017 (for which she will be workshops chair). She holds an MSc degree from MIT (2000) and a PhD in Computer Science from UFR] (2007).

Ana Cristina B. Garcia is a full professor at Universidade Federal Fluminense since 2010. She is also, since 1996, the founder and general coordinator of ADDLabs, a R\&D lab in Artificial Intelligence and Human-Computer Interaction with great focus on solving problems for the Oil\&Gas industry. She has published over 26 journal papers, 2 edited books, 12 book chapters and 148 conference papers. She also advised 7 Ph.D. and 28 M.Sc. students. Her current research interests are artificial intelligence, collective intelligence, ambient intelligence and human-computer interaction. She graduated in Civil Engineering from Universidade Federal do Rio de Janeiro (1983), M.Sc. (1989) and Ph.D. (1992) from Stanford University.

José H. Canós is an associate professor at the Department of Computer Science (DSIC) of the Universitat Politècnica de València, Spain, where he leads the Software Engineering and Information Systems Research Group. He holds a degree in Physics from the University of Valencia (1984) and a Ph.D. in Computer Science from the Universitat Politècnica de 
Valencia (1996). His current research interests are Digital Libraries, Document Engineering and Emergency Management Information Systems. He has participated in national, European and Iberoamerican research projects. He was the Program Chair of the 20th Spanish Conference on Software Engineering and Databases (JISBD 2015).

Tina Comes is Full Professor in the Department of ICT, University of Agder, Norway, Deputy Director of the Centre for Integrated Emergency Management. Dr Comes holds a position as Senior Researcher at the Smart Instrumentation Group with Teknova AS, and is currently a fellow at the Harvard Humanitarian

Initiative and the Harvard Kennedy School. She studied Mathematics, literature and philosophy. She received her Ph.D. from the Karlsruhe Institute of Technology. Her research aims at supporting decision-making and risk management in complex, dynamic and uncertain situations. She focuses on the development of collaborative and distributed decision support tools, which are designed to facilitate coordination and fast adaptation in dynamic environments. Dr. Comes is author of numerous papers published in international journals and conferences, and she has been actively promoting the topic of decision support in emergency management. She was Program Chair for ISCRAM2013, Conference Chair for ISCRAM2015 and is currently Vice President of the ISCRAM Association.

Vaninha Vieira is Associate Professor in the Computer Science Department, UFBA, and researcher of the Software Engineering Lab (LES). She is the Scientific Director of the Fraunhofer Project Center for Software and Systems Engineering at UFBA (FPC-UFBA), and coordinator of the CEManTIKA research group on mobile, ubiquitous and context-sensitive systems. She just returned from a sabbatical license (post-doc) at the University of Southern California (USC) (2015). She received a Ph.D. in Computer Science from the Federal University of Pernambuco (2008), with one-year internship in University Paris VI, France (2007). She has a Master Degree in Computer Science from the Federal University of Rio de Janeiro - COPPE/UFRJ (2003) and graduated in Computer Science from Federal University of Bahia (1999). She has academic and professional experience in Computer Science, with special emphasis in Databases and Software

Engineering. Her research interests include ContextAware Computing, Mobile and Ubiquitous Computing, Collaborative Systems and Smart Cities, with emphasis in the domains of Crisis and Emergency Management, and Intelligent Transportation Systems.

\section{Acknowledgements}

Adriana S Vivacqua is partially supported by $\mathrm{CNPq}$ grant 308425/2012-0 and FAPERJ grant E$26 / 103.029 / 2012$. Vaninha Vieira is partially supported by CNPq grant 490084/2013-3.

\section{References}

1. Eide, A.W., Haugstveit, I.M., Halvorsrud, R., Skjetne, J.H., Stiso, M. Key Challenges in Multiagency Collaboration during Large-scale Emergency Management. In: Proceedings of the Workshop on Ambient Intelligence for Crisis Management. Pisa Italy, 2012. http://ceur-ws.org/Vol-953/

2. Haarmann, S. Podlesny, N.J., Hewelt, M., Meyer, A. Weske, M. Production Case Management: A Prototypical Process Engine to Execute Flexible Business Processes. In: Proceedings of the BPM Demo Session 2015, 13th International Conference on Business Process Management (BPM 2015), 
CEUR vol.1418, 2015. http://ceur-ws.org/Vol$1418 /$

3. Herzberg, N., Kirchner, K., Weske, M. Modeling and Monitoring Variability in Hospital Treatments: A

Scenario Using CMMN. In: Business Process

Management Workshops. Lecture Notes in Business Information Processing vol 202 pp 3-15, Springer, 2015

4. Kapucu, N., Garayev, V. Collaborative DecisionMaking in Emergency and Disaster Management. International Journal of Public Administration, 34: 366-375, 2011

5. Kendra, J., Wachtendorf, T. Improvisation, Creativity, and the Art of Emergency Management. In: Understanding and Responding to Terrorism, $\mathrm{P}$ 324-335, Huseyin Durmaz, Bilal Sevinc, et al., eds. IOSPress, Netherlands, 2007

6. Kendra, J.M., Wachtendorf, T. Elements of Resilience After the World Trade Center Disaster: Reconstituting New York City's Emergency

Operations Centre. In Disasters, 2003, 27(1): 3753

7. Mendonça, D., Beroggi, G.E.G., Wallace, W.A Decision support for improvisation during emergency response operations. In: International Journal on Emergency Management, Vol. 1, No. 1, 2001

8. Mendonça, D., Jefferson, T., Harrald, J.

Collaborative adhocracies and mix-and-match technologies in emergency management. In: Communications of the ACM, Vol.50, No.3, pp 4449, ACM New York, 2007

9. Waugh Jr., W.L., Streib, G. Collaboration and Leadership for Effective Emergency Management. In Public Administration Review, Vol. 66, Issue Supplement s1, pp. 131-140, December 2006. 